\title{
NMR Study of $\beta$ Relaxation in Modified and Blended Isotactic Polypropylene
}

\author{
Dušan OlČÁK, ${ }^{*}$ Ladislav ŠEvČOvič, ${ }^{*}$ L'ubomír MuCHA, ${ }^{*}$ and Ol'ga ĎURČOvÁ** \\ * Department of Physics, Technical University, 04120 Košice, Slovak Republic \\ ** Research Institute of Man-Made Fibres, 05921 Svit, Slovak Republic
}

(Received June 23, 1995)

\begin{abstract}
Broad line NMR and spin-lattice relaxation times $T_{1}$ have been used to study glass transition phenomena in isotactic polypropylene modified and blended with an ethylene aminoalkylacrylate copolymer. By means of decomposition of spectra into elementary components corresponding to the chains with different mobility a softening due to the polymer modifier was observed. The modification results in an increase of fractions of amorphous and intermediate chains, respectively, gives rise to the enhancement of the chain mobility in amorphous regions, but the higher amount of the polymer modifier, which was used in the range up to $15 \mathrm{wt} \%$, the lower chain mobility in intermediate regions was found out. From the temperature dependences of the spin-lattice relaxation times $T_{1}$ measured over the temperature range $290-430 \mathrm{~K}$, a broadening of correlation time distribution on the side of the shorter correlation times has been found out to be and it was ascribed to overlapping of relaxation processes in amorphous regions. The sample containing the highest amount of the polymer modifier, in contrast to others, was shown to blend not only on the molecular level but also on the overmolecular.

KEY WORDS Modified and Blended Polypropylene / Glass Transition / Broad Line Nuclear Magnetic Resonance (NMR) / Spin-Lattice Relaxation Times / Correlation Time Distribution /
\end{abstract}

In many of the previous works it was shown that isotactic polypropylene (PP) has a complex structure with regions of the different chain arrangement and different chain mobility. A heterogeneity, which may be studied by means of the broad line NMR spectra ${ }^{1-7}$ (ref 1 is to see the earlier papers) gives rise to the multiple transitions occuring in the temperature range around and above the glass transition of PP. ${ }^{8}$ Three components of the rotating frame spin-lattice relaxation times $T_{1 \rho}$ with several relaxation minima in their temperature dependences also confirmed such relaxation behaviour. ${ }^{9,10}$ On the other hand, due to spin diffusion only one relaxation time $T_{1}$, a value of which is obviously determined by the most quickly relaxing, i.e., amorphous chains, may usually be measured. The minimum being appeared in the temperature range above the glass transition of PP corresponds to the $\beta$ relaxation which was assigned to the glass transition phenomena..$^{9,11-15}$

In the precedent paper a considerable effect of the polymer modifier PFDA 522 on relaxation processes in PP was found out by the rotating frame spin-lattice relaxation times $T_{1 \rho} \cdot{ }^{10}$ By the modification two temperature shifts towards the low temperatures were provoked in the amorphous regions of modified PP. They were the small shift only of the $\beta$ relaxation corresponding to the segmental motion and the marked shift of the $\alpha_{\mathrm{a}}$ process, which was ascribed to the motion of the whole amorphous chains. This behaviour produced an overlapping of both discussed relaxations. It is reasonable to expect that this effect will also be observable by the spin-lattice relaxation times $T_{1}$. But on the score of the spin diffusion only averaged parameters can be drawn from the $T_{1}$ measurements, an interpretation of which is not always unambiguos. On the other hand, it turns out that the broad line NMR spectra provide data helpful to interpret the temperature dependences of the relaxation times $T_{1}$. Because of these reasons the study of the $\beta$ relaxation on modified PP by means of the broad line NMR spectra and spin-lattice relaxation times $T_{1}$ has been undertaken.

\section{EXPERIMENTAL}

The ${ }^{1} \mathrm{H}$ NMR measurements were carried out on the granulated samples of PP Mosten D3, ${ }^{10}$ polymer modifier PFDA 522 which consists primarily of an ethylene aminoalkylacrylate copolymer ${ }^{16,17}$ and on the modified PP samples which are materials of PP blended with the different amounts of the polymer modifier. ${ }^{10}$ By the weight percentage of PFDA 522 the modified samples are designated as PP-X, where $X=2,4,6,8,10$, and 15 . However, according to the Pennisson nomenclature $^{18}$ only the samples with $X=2-10$ are samples of modified PP, and PP- 15 containing more than $10 \mathrm{wt} \%$ of each of both components is a polymer blend. This classification will be respected only in inevitable cases, otherwise all these samples will be denoted as modified. No treatment of unmodified PP was made and this sample is denoted as PP-0.

Broad line NMR spectra and spin-lattice relaxation times $T_{1}$ were measured with the continuous wave and the pulse spectrometer, both constructed in our laboratory. ${ }^{19,20}$ The former operates using the frequency sweeping at the fixed magnetic field $B_{0}=0.247 \mathrm{~T}\left(f_{0}=\right.$ $10.5 \mathrm{MHz}$ ). Frequency sweep and data acquisition were made by means of the personal computer. In the units of the magnetic field induction the sweep range is $5 \mathrm{mT}$ and in this range the NMR lines were recorded by 1024 points. All spectra were detected at the temperature $302 \mathrm{~K}$ and the same operating parameters of the spectrometer, with the exception of those for PFDA 522, were used. The frequency sweep, amplitude and frequency of the modulating field and time constant of the lock-in amplifier were $1.39 \mu \mathrm{Ts}^{-1}, 46 \mu \mathrm{T}, 40 \mathrm{~Hz}$, and $0.12 \mathrm{~s}$, respectively. The modulating field amplitude for PFDA 522 was $22 \mu \mathrm{T}$. To improve signal to noise ratio about 15 scannings were accumulated.

Spin-lattice relaxation times $T_{1}$ were measured by the saturation technique ${ }^{21}$ using $90_{\text {sat }}^{\circ}-\tau-90^{\circ}$ pulse sequence at the resonant frequency $f_{0}=30 \mathrm{MHz}$ over the temperature range from 290 to $430 \mathrm{~K}$. All relaxation 
curves except for those measured within the narrow temperature range close to the room temperature were exponential, and to achieve a higher accuracy they were established from ten accumulations. The errors of the measurements were found out to be less than $3.0 \%$. During measurements, the set temperature was kept with an accuracy $\pm 0.1 \mathrm{~K}$.

\section{RESULTS AND DISCUSSION}

\section{Broad Line NMR}

A structure of partially crystalline PP is frequently interpreted as the three phase system with amorphous, intermediate and crystalline phase. ${ }^{1,7,8,22-28}$ In depen- dence on the molecular motions activated within individual phases, broad line NMR spectra can be decomposed into several components. The well known method of decomposition elaborated by Bergmann gives the results which show an agreement with other techniques. ${ }^{22,29,30}$ According to this method the NMR spectrum can be decomposed into three elementary spectra $y_{\mathrm{n}}$ (narrow), $y_{\mathrm{m}}$ (middle), $y_{\mathrm{b}}$ (broad) corresponding to the chains with different mobility. Usually, the narrow component $y_{\mathrm{n}}$ is the Lorentzian function, the middle $y_{\mathrm{m}}$ is the product of the Gaussian and the Lorentzian function and the broad elementary spectrum is derived from the low temperature spectrum. Other possibilities were also verified. ${ }^{31}$

The spectra shown in Figure 1 make it possible to

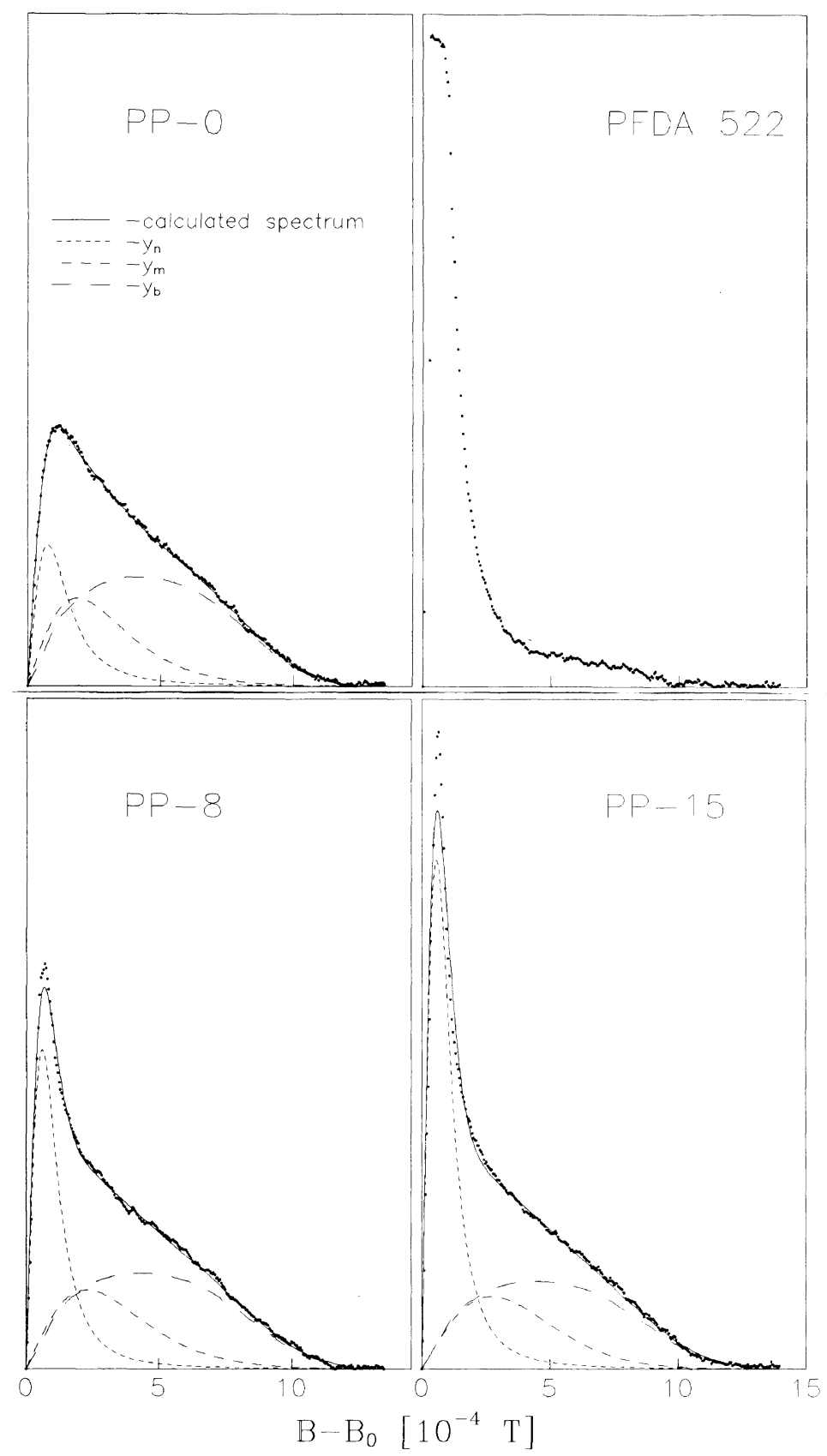

Figure 1. Derivations of the broad line n.m.r. absorptions measured on the polymer modifier PFDA 522 and on the samples of unmodified (PP-0) and modified (PP-8, PP-15) PP. Because of the very intensive narrow (amorphous) peak and very weak broad (crystalline) line it was not possible to draw the complete spectrum of PFDA 522 at $302 \mathrm{~K}$. All spectra besides that of PFDA 522 are normalized. The decomposition into elementary spectra $y_{\mathrm{b}}, y_{\mathrm{m}}$, and $y_{\mathrm{b}}$ with the least square method is shown. It was found out that the low temperature spectra measured on the samples PP- $X$ $(X=0-15)$ do not differ each other, therefore, in all cases the low temperature spectrum measured on PP-8 at $193 \mathrm{~K}$ was used to derive the broad components $y_{\mathrm{b}}$. The effect of the modulation amplitude on the narrow component $y_{\mathrm{n}}$ was considered in the computations. 


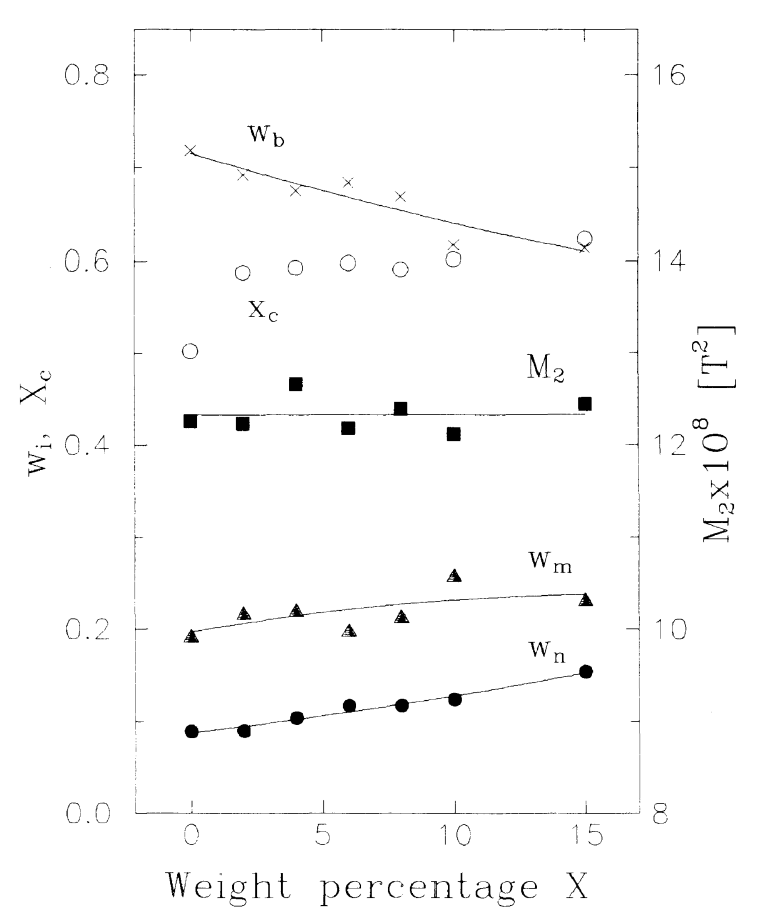

Figure 2. The dependence of the second moment $M_{2}$, mass fractions $w_{i}(i=\mathrm{n}, \mathrm{m}, \mathrm{b})$ and crystallinity $X_{\mathrm{c}}$ on the percentage $X$ of the polymer modifier PFDA 522 in the unmodified and modified samples PP- $X$.

understand the effect of the modification. The spectrum of the polymer modifier which consists primarily of the very intensive narrow line reveals both the predominantly amorphous character of the sample and the high mobility of the amorphous chains. The nature of this polymer is also reflected in the spectra of the modified samples where the polymer modifier has produced the obvious changes of their shape, and the higer amount of the polymer modifier the more intensive narrow peak in the spectra of modified PP was observed. On the other hand, there was not observed an influence of these changes on the second moment $M_{2}$ and, as it can be seen in Figure 2, it takes on the values from the narrow interval $0.121-0.127 \mathrm{mT}^{2}$. An explanation of this disagreement provides the decomposition of the spectra into the elementary components.

The dependences of the mass fractions $w_{\mathrm{n}}, w_{\mathrm{m}}, w_{\mathrm{b}}$ and the crystallinity $X_{\mathrm{c}}$ on the percentage of the polymer modifier are shown in Figure 2 . The values of the crystallinity of all samples besides that of PP-0 are close to 0.60 . The difference in the $X_{\mathrm{c}}$ between unrecrystallised sample PP-0 and other samples can be explained rather by the different thermal history than by blending. There are also differences between the mass fractions $w_{\mathrm{b}}$ of the rigid chains and the crystallinities $X_{\mathrm{c}}$. The values of the $w_{\mathrm{b}}$ show a decrease with the increase of the amount of the polymer modifier and gradually approach to the values of the crystallinity. Therefore, with the decomposition procedure a softening may allow us an easier separation of the noncrystalline chains from crystalline. In contrast with the $w_{\mathrm{b}}$, both the mass fraction $w_{\mathrm{n}}$ of the high mobile, amorphous chains and the mass fraction $w_{\mathrm{m}}$ of the hindered mobile, intermediate chains exhibit the increase in the dependence in Figure 2.

Width and shape of the individual elementary spectra are characterized by their parameters $\beta_{\mathrm{L}}, \beta_{\mathrm{mL}}, \beta_{\mathrm{mG}}$, and

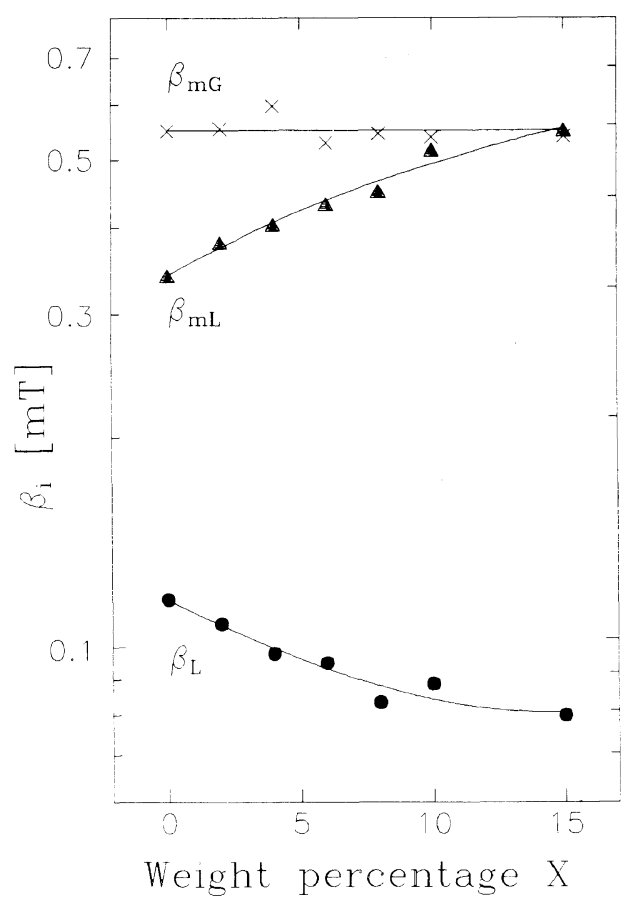

Figure 3. The dependences of the parameters $\beta_{i}(i=\mathrm{L}, \mathrm{mL}, \mathrm{mG})$ connected with the particular elementary spectra on the percentage $X$.

$s$. The scaling parameter $s$ derived from the low temperature spectrum and connected with the broad component varies only within the small interval $0.950-0.970$ and so it can be regarded as a constant. The parameter $\beta_{\mathbf{L}}$ connected with the narrow component reflects the chain mobility in the amorphous regions and its decrease, which can be seen in Figure 3, reveals the mobility enhancement of the amorphous chains in modified PP. By means of the parameters $\beta_{\mathrm{mL}}$ and $\beta_{\mathrm{mG}}$ the width and shape of the middle component, which we have attributed to the intermediate regions, are described. The $\beta_{\mathrm{mG}}$ parameter is independent on the percentage $X$ over the whole range $0-15 \mathrm{wt} \%$, however, the parameter $\beta_{\mathrm{mL}}$ shows a distinct rise in this dependence. Such behaviour of the parameters $\beta_{\mathrm{mL}}$ and $\beta_{\mathrm{mG}}$ is on the score of the decreasing mobility of the intermediate chains of modified PP. So, opposite effects of the polymer modifier in amorphous and intermediate regions were observed, which contribute to the maintance of the constant value of the second moment $M_{2}$. To confirm this statement the computations of the second moment $M_{2}$ of the elementary spectra were made and the second moments of the narrow, middle and broad components, for instance for PP-0 and PP-10 are 0.61, 0.72, 1.44 and 0.47, $0.90,1.49 \mathrm{mT}^{2}$, respectively. The decrease of the second moment of the narrow line and on the contrary the increase of the second moment of the middle line is seen from the data. The sums of these data weighted by the corresponding mass fractions yield the second moments $M_{2}$ of the samples. Therefore, from the results mentioned above it may be concluded that the modifier interacts with the chains of noncrystalline regions, whereby the rigid chains of noncrystalline regions are converted onto the hindered mobile, but their mobility is lower as that of the unmodified PP chains. Both, the higher mobility and the increase of the amorphous mass fraction were observed to be provoked by the modifica- 
tion.

\section{Spin-Lattice Relaxation Times}

For an interpretation of the temperature dependences of the spin-lattice relaxation times $T_{1}$ measured on polymers, the elementary theory of the nuclear magnetic relaxation is usually employed. ${ }^{32}$ Conditions for the minimum together with the Arrhenius law provide important parameters which reflect a molecular dynamics of the polymer under study. To account for a distribution of the correlation times $\tau_{\mathrm{c}}$ a choice of the several well known distribution functions can be made. ${ }^{33,34}$ The last appeared, modified Fuoss-Kirkwood (mFK) distribution, ${ }^{35,36}$ particularly suitable for an account of the temperature dependences of the $T_{1}$ with different slopes on the low and high temperature sides of the minimum, was considered in our calculation. Using the mFK distribution the dependence of the spin-lattice relaxation time $T_{1}$ on the correlation time $\tau$, characteristic for the distribution $\mathrm{g}\left(\tau_{\mathrm{c}}\right)$ under consideration, is expressed by the equation

$$
\frac{1}{T_{1}}=\frac{C}{\omega} \frac{\alpha+\beta}{2} \sin \frac{\alpha \pi}{\alpha+\beta}\left[\frac{(\omega \tau)^{\alpha}}{1+(\omega \tau)^{\alpha+\beta}}+2 \frac{(2 \omega \tau)^{\alpha}}{1+(2 \omega \tau)^{\alpha+\beta}}\right]
$$

in which $C$ is the relaxation constant, $\omega$ is the circular resonant frequency and $\alpha$ and $\beta$ are parameters of the mFK distribution. The spin-lattice relaxation times take on the minimum $T_{1, \min }$ when the condition $(\omega \tau)(\beta / \alpha)^{1 /(\alpha+\beta)}=$ 0.6158 is fulfilled. ${ }^{36}$ If the correlation time $\tau$ is expressed by means of the Arrhenius law $\tau=\tau_{0} \exp \left(E_{\mathrm{a}} / R T\right)$, then the approach mentioned above enables the relaxation constant $C$, parameters $\alpha$ and $\beta$, preexponential factor $\tau_{0}$ and activation energy $E_{\mathrm{a}}$ to be drawn from the measured temperature dependences of the spin-lattice relaxation times $T_{1}$.

In accordance with the conclusions made from the broad line NMR the temperature dependences of the $T_{1}$ are predicted not to be very changed by the modification.

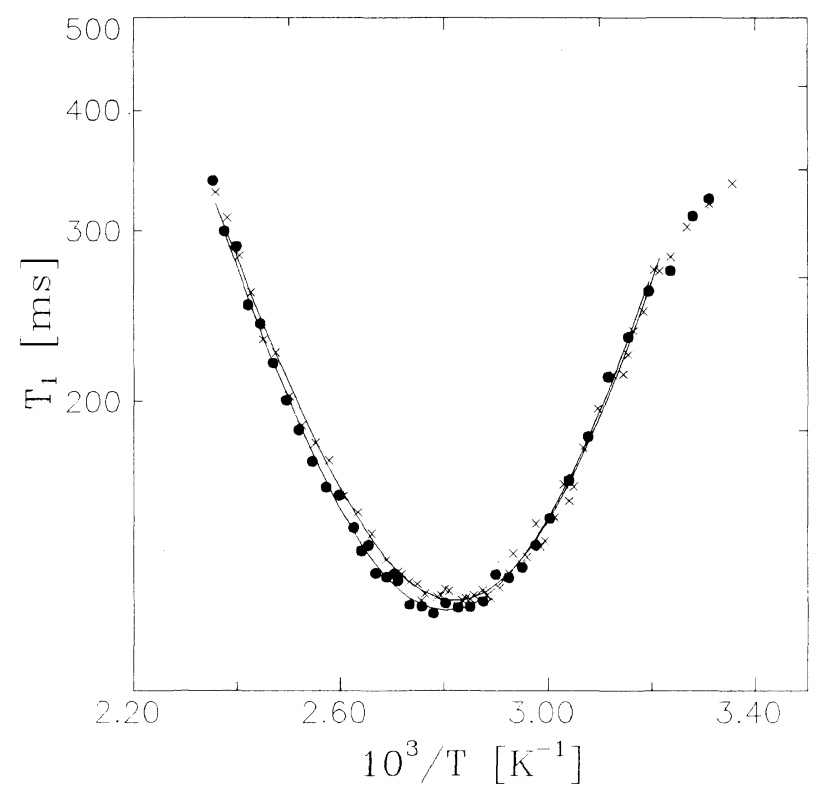

Figure 4. The temperature dependences of the spin-lattice relaxation times $T_{1}$ measured on the samples PP-2 (O) and PP-8 $(\times)$. The solid lines represent the dependences obtained by means of the fitting procedure.
The dependences measured on the samples PP-2 and PP- 8 of modified PP depicted in Figure 4 confirm this assumption. The minima corresponding to modified PP are shown in Table I to be slightly shifted towards the lower temperatures and their values $T_{1, \min }$ are higher than that of unmodified PP. The increase of the $T_{1, \min }$ can be interpreted in terms of the higher crystallinity and spin diffusion effects, ${ }^{15,37,38}$ but it can also result from the larger distribution of the correlation times $\tau_{\mathrm{c}} \cdot{ }^{35,36,39}$

The relaxation time $T_{1, \text { min }}$ as well as the relaxation constant $C$ are associated with the part of the rigid lattice second moment $\Delta M_{2, \mathrm{rl}}$ which is averaged by the molecular process under consideration, and the proportionalities $1 / T_{1, \min } \sim C \sim \Delta M_{2, \mathrm{rl}}$ are valid. ${ }^{40}$ The rise of the $C$ depending on the increasing percentage of the polymer modifier (Table II) should lead to the decrease of the relaxation times $T_{1, \min }$. However, according to the $T_{1, \min }$ values of the modified samples introduced in Table I, the phenomena resulting in the decrease of the $T_{1, \min }$ are shown to be overcomed by the phenomena which give rise to its increase. The relaxation time $T_{1, \min }$ unlike the constant $C$ is also dependent on the distribution of the correlation times $\tau_{\mathrm{c}}$. Therefore, a disagreement between behaviours of the $C$ and of the $T_{1, \min }$ can be explained by the broadening of the correlation time distribution. The crystallinities of the modified samples $(X=2-15)$ show unessential variations, therefore the influence of the spin diffusion will not be decisive. ${ }^{15}$

A substantial change was observed in the correlation time distribution which is characterized by the $\alpha$ and $\beta$ parameters of the modified Fuoss-Kirkwood distribution. From the values of $\alpha$ and $\beta$ it may be noted that in the case of the modified sample PP-2 the correlation time spectrum is the narrowest and shows only a weak asymmetry. Moreover, from the dependence of the parameter $\alpha$ we conclude that the correlation time spectrum broadens on the side of the shorter correlation times, while on the side of the longer characterized by the parameter $\beta$, the spectrum is practically independent on

Table I. The minima positions $T_{\min }, T_{1, \min }$ of the temperature dependences of the laboratory frame spin-lattice relaxation $T_{1}$ measured over the temperature range of $\beta$ relaxation

\begin{tabular}{lccc}
\hline Sample & $X_{\mathrm{c}}^{\mathrm{a}}$ & $T_{\min } / \mathrm{K}$ & $T_{1, \min } / \mathrm{ms}$ \\
\hline PP-0 & 0.502 & 356.2 & 152 \\
PP-2 & 0.588 & 356.6 & 155 \\
PP-4 & 0.593 & 355.9 & 156 \\
PP-6 & 0.598 & 355.9 & 159 \\
PP-8 & 0.591 & 354.4 & 159 \\
PP-15 & 0.623 & 353.5 & 162 \\
\hline
\end{tabular}

${ }^{\mathrm{a}} X_{\mathrm{c}}, \mathrm{X}$-ray crystallinities of the samples.

Table II. Fitting data of the $\beta$ relaxation parameters obtained from the temperature dependences of the spin-lattice relaxation times $T_{1}$

\begin{tabular}{lccccc}
\hline Sample & $C \times 10^{-8} / \mathrm{s}^{-2}$ & $\alpha$ & $\beta$ & $\tau_{0} \times 10^{14}$ & $E_{\mathrm{a}} / \mathrm{kJ} \mathrm{mol}^{-1}$ \\
\hline PP-0 & 9.50 & 0.88 & 0.94 & 3.42 & 33.9 \\
PP-2 & 8.75 & 0.95 & 1.00 & 6.53 & 32.0 \\
PP-4 & 9.12 & 0.87 & 0.99 & 4.07 & 33.2 \\
PP-6 & 9.56 & 0.82 & 0.90 & 2.32 & 34.9 \\
PP-8 & 9.86 & 0.77 & 0.94 & 1.65 & 35.6 \\
PP-15 & 9.86 & 0.72 & 0.93 & 1.41 & 35.9 \\
\hline
\end{tabular}




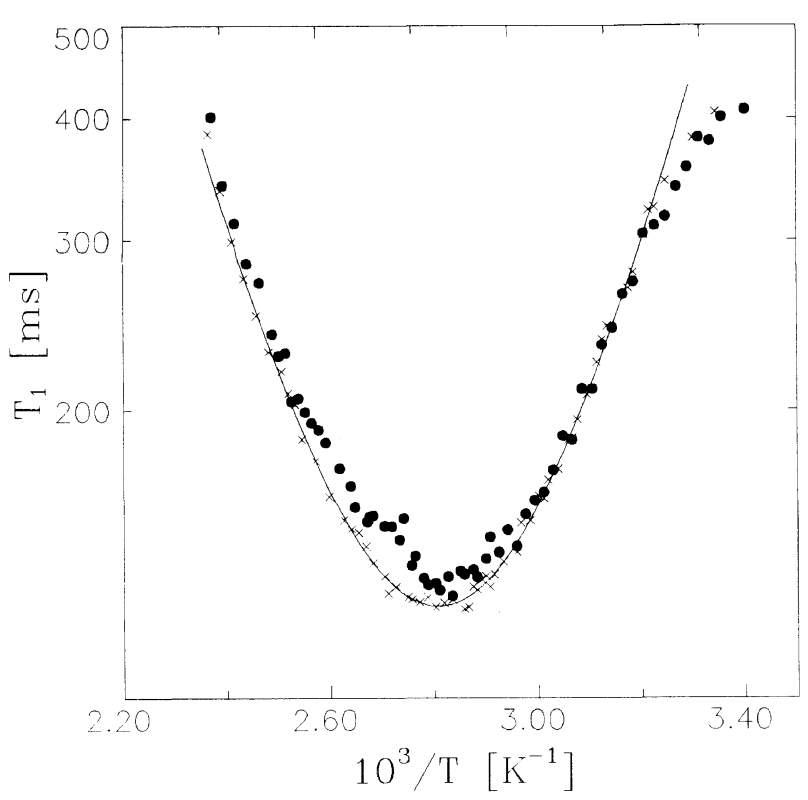

Figure 5. The temperature dependences of the spin-lattice relaxation times $T_{1}$ measured on the samples PP-0 $(\times)$ and PP-15

the amount of the polymer modifier PFDA 522. We assume that the change of the spectrum on the side of the short correlation times arises from the shift of the $\alpha_{\mathrm{a}}$ relaxation process towards the lower temperatures and its partial overlapping with the $\beta$ relaxation process. Such effect was revealed on PP-8 by means of the rotating frame spin-lattice relaxation times $T_{1 \rho} \cdot{ }^{10}$ So, the temperature dependences of the spin-lattice relaxation times $T_{1}$ include not only segmental motion but gradually with the increasing amount of the polymer modifier also the free reorientation of the whole chains of the amorphous regions.

As we have mentioned previously, the modification results in the increase of the fractions corresponding to the narrow and middle component, gives rise to the enhancement of the chain mobility in the amorphous regions, but the higher amount of the polymer modifier the lower chain mobility was observed in the intermediate regions. Obviously, the apparent activation energy is determined by the motions in both discussed regions and its increase in Table II can be explained by dominating effect of the intermediate chains.

A preexponential factor $\tau_{0}$ may be interpreted in terms of the chain arrangement in noncrystalline regions. It may be expressed by the relation $\tau_{0}=A_{\tau} \exp \left(-\Delta S_{\mathrm{a}} / R\right)$ in which $A_{\tau}$ is a characteristic time constant of the motion and $S_{\mathrm{a}}$ is activation entropy. ${ }^{41}$ A change of the activation entropy $\Delta S_{\mathrm{a}}$ of the ensemble of the relaxing chains owing to the decrease of their arrangement provides explanation of the decrease of the $\tau_{0}$ (Table II) at the simultaneous increase of the apparent activation energy $E_{\mathrm{a}}$.

The $T_{1}$ temperature dependence for PP-15, in the contrary to the others, is narrowed at the region closed to the minimum and a shoulder is appeared at the high temperature side of this dependence at $365-377 \mathrm{~K}$ (Figure 5). We believe the relaxation process detected by this manner is connected with the melting of PFDA 522 in polymer blend PP-15. Both, the melting temperature which is $360 \mathrm{~K}^{10}$ and the $T_{1}$ measurements on PFDA

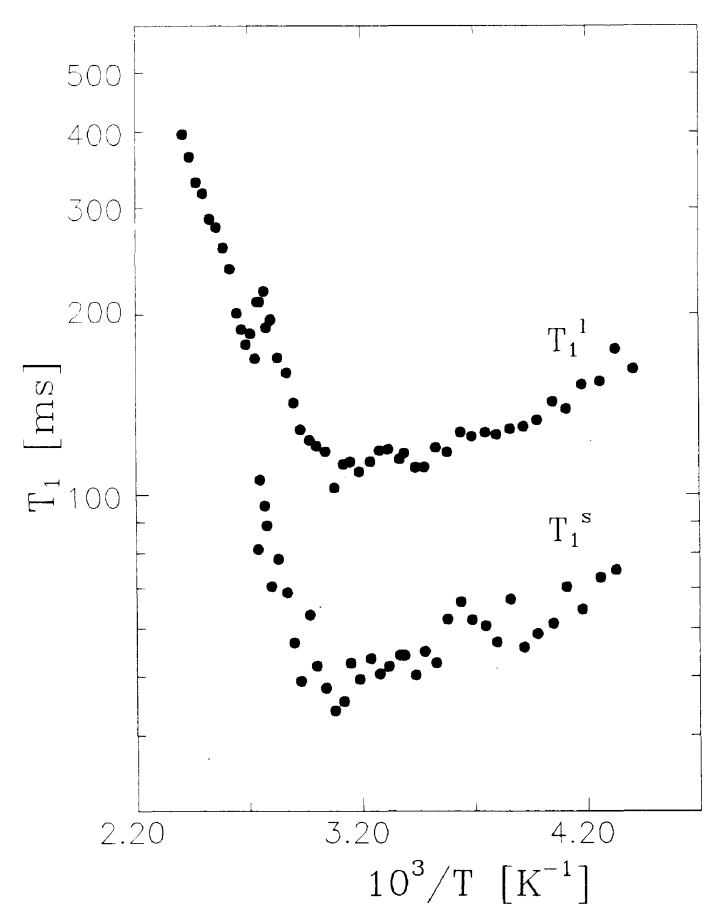

Figure 6. The temperature dependences of the spin-lattice relation times $T_{1}$ measured on the polymer modifier PFDA 522 in the temperature range $225-415 \mathrm{~K}$. Relaxation curves detected at the temperatures which are lower than $365 \mathrm{~K}$ give two relaxation times $T_{1}{ }^{1}$ (long) and $T_{1}{ }^{\mathrm{s}}$ (short). Above $365 \mathrm{~K}$ only one spin-lattice relaxation time $T_{1}$ can be observed with the minimum appeared at $370 \mathrm{~K}$.

522 (Figure 6) confirm our assumption. Two relaxation times $T_{1}$ where observed on PFDA 522 within the broad temperature range. One of them gradually disappears with the increasing temperature and remaining shows the minimum at $370 \mathrm{~K}$. It is reasonable to assume that these effects reflect the melting process and the minimum mentioned above produces the shoulder in the $T_{1}$ temperature dependence for the blend PP-15. From this we can conclude that domains of the pure modifier PFDA 522 must be present in the matrix of PP to observe its melting in the polymer blend PP-15. But, as it results from the analysis of the relaxation behaviour of the modified samples, the blending on the molecular level was also observed in PP-15.

\section{REFERENCES}

1. H. Tanaka, J. Appl. Polym. Sci., 27, 2197 (1982).

2. J. Murín, D. Olčák, M. Rákoš, and R. Šimo, Acta Phys. Slov., 31, 229 (1981).

3. E. A. Egorov and V. V. Zhizhenkov, J. Polym. Sci., Polym. Phys. Ed., 20, 1089 (1982).

4. K. J. Packer, J. M. Pope, R. R. Yeung, and M. E. A. Cudby, J. Polym. Sci., Polym. Phys. Ed., 22, 589 (1984)

5. J. Murín and D. Olčák, Czech. J. Phys., B34, 247 (1984).

6. A. M. Kenwright, K. J. Packer, and B. J. Say, J. Magn. Reson., 69, 426 (1986).

7. Z. Špaldonová and D. Olčák, Acta Phys. Slov., 42, 74 (1992).

8. M. Jarrigeon, B. Chabert, D. Chatain, C. Lacabanne, and G. Nemoz, J. Macromol. Sci. Phys., B17, 1 (1980).

9. D. Olčák, J. Murín, J. Uhrin, M. Rákoš, and W. Schenk, Polymer, 26, 1455 (1985).

10. D. Olčák, A. Stančáková, Z. Špaldonová, and O. Katreniaková, Polymer, 36, 487 (1995).

11. T. Kawai and A. Hirai, J. Phys. Soc. Jpn., 16, 2356 (1961).

12. U. Kienzle, F. Noack, and J. von Schütz, Kolloid Z.-Z. Polym., 326, 129 (1970). 
13. V. J. McBrierty, D. C. Douglass, and D. C. Falcone, J. Chem. Soc., Faraday Trans. 2., 68, 1051 (1972).

14. H. Tanaka and K. Saito, Colloid Polym. Sci., 266, 1 (1988).

15. J. Murín, D. Geschke, P. Holstein, D. Olčák, L. Ševčovič, and L'. Mucha, Acta Polymerica, 39, 389 (1988).

16. Sumitomo Chemical, Co., Jpn. Patent No. 22523 (1967).

17. T. Ohmae and S. Abeta, "Dyeable Modifier and Dyeing of Polyolefin Fibers," in "14th Conference on Fibre-Forming Polymers and Their Treatment," Štrbské Pleso (Slovakia), 1981; Czechoslovak Scientific and Technical Society, Research Institute of Man-Made Fibres, Svit; Dom Techniky, Košice (Slovakia) 1981

18. R. Pennisson, "Proceedings, 12th International Conference on Man-Made Fibres," Dornbirn, Leuziger Ber. Nr. 36, 1974, p 24.

19. L. Ševčovič and F. Mede, J. Electrical Eng., 42, 584 (1991).

20. L. Ševčovič and J. Lašanda, J. Proc. Sci. Works Techn. Univ. Košice, 3, 89 (1989) (Abstract in English).

21. E. Fukushima and S. B. W. Roeder, "Experimental Pulse NMR," Addison-Wesley Publishing Company, Inc., Reading, Massachusetts, 1981, p 174.

22. K. Bergmann, J. Kolloid. Z. Z. Polym., 251, 962 (1973)

23. H. Tanaka, Colloid Polym. Sci., 260, 1101 (1982).

24. M. Ito, H. Serizawa, K. Tanaka, W. P. Leung, and C. L. Choy, J. Polym. Sci., Polym. Phys. Ed., 21, 2299 (1983).
25. H. Tanaka, J. Appl. Polym. Sci., 28, 1707 (1983).

26. H. Tanaka, Eur. Polym. J., 21, 673 (1985).

27. M. Ito and K. Tanaka, Polym. J., 18, 557 (1986).

28. H. Tanaka, F. Kohrogi, and K. Suzuki, Eur. Polym. J., 25, 449 (1989).

29. K. Bergmann, J. Polym. Sci., Polym. Phys. Ed., 16, 1611 (1978).

30. J. Uhrin, J. Murin, and D. Olčák, Acta Phys. Slov., 34, 209 (1984).

31. K. Unterforshuber and K. Bergmann, J. Magn. Reson., 33, 483 (1979).

32. N. Bloembergen, E. M. Purcell, and R. V. Pound, Phys. Rev., 73, 679 (1948)

33. V. J. McBrierty, Magn. Reson. Rev., 8, 165 (1983)

34. P. A. Beckmann, Physics Reports (Review Section of Physics Letters), 171, 85 (1988)

35. V. D. Fedotov and H. Schneider, NMR: Basic Princ. Prog., 21, 1 (1989).

36. H. Schneider, J. Polym. Sci., Polym. Phys. Ed., 29, 1171 (1991).

37. V. J. McBrierty, Polymer, 15, 503 (1974).

38. H. Tanaka and K. Takagi, Eur. Polym. J., 26, 153 (1990).

39. T. Kawai, Y. Yoshimiu, and A. Hirai, J. Phys. Soc. Jpn., 16, $2356(1991)$

40. G. Hempel and H. Schneider, Polym. Bull., 6, 7 (1981).

41. G. M. Bartenev and S. Ja. Frenkel, "Fizika Polimerov," Chimija, Leningrad, 1990, p 174 